\title{
Herausgeber:
}

Prof. Dr. Klaus Boers, Universität Münster | Prof. Dr. Heinz Cornel, Alice Salomon-FH Berlin | Prof. Dr. Frieder Dünkel, Universität Greifswald | Prof. Dr. Monika Frommel, Universität Kiel | Prof. Dr. Wolfgang Heinz, Universität Konstanz | Prof. Gabriele Kawamura-Reindl, Georg-SimonOhm-FH Nürnberg | Prof. Dr. Joachim Kersten, Deutsche Hochschule der Polizei, Münster | Prof. Dr. Karl-Ludwig Kunz, Institut für Strafrecht und Kriminologie, Bern | Prof. Dr. Heribert Ostendorf, Forschungsstelle für Jugendstrafrecht und Kriminalprävention an der Universität Kiel | Prof. Dr. Bernd-R. Sonnen, Universität Hamburg | PD Dr. Wolfgang Stangl, Institut für Rechts- und Kriminalsoziologie, Wien | Dr. Joachim Walter, JVA Adelsheim

\section{Reformgesetz zu „Familiengerichtlichen Maßnahmen bei Gefährdung des Kindeswohls“ in Kraft getreten!}

Frieder Dünkel

Gravierende Fälle von Kinder- und Jugenddelinquenz sowie der Vernachlässigung und Misshandlung von Kindern hatten in der Vergangenheit zu Überlegungen geführt, einerseits das Jugendstrafrecht zu verschärfen (vgl. die von einigen Politikern immer wieder in die Diskussion gebrachte Herabsetzung des Strafmündigkeitsalters auf 12 Jahre) und andererseits im Bereich des Jugendhilferechts die Interventionsmöglichkeiten gegenüber Kindern und Jugendlichen sowie den Eltern auszuweiten. Die aktuellen Tendenzen der Jugendpolitik wie sie in dem Abschlussbericht einer unter Federführung des Bundesjustizministeriums gebildeten Arbeitsgruppe von Experten eindeutig zum Ausdruck gelangten (s. u.), heben sich in erfreulicher Weise von Verschärfungstendenzen der vergangenen Jahre ab (vgl. den Abschlussbericht vom 17.11.2006, der in dem am 12.7.2008 in Kraft getretenen Gesetz, BGBl 2008, Teil I, Nr. 28, S. 1188 ff., nahezu vollständig umgesetzt wurde). Sie lassen sich wie folgt zusammenfassen:

- $\quad$ Eine Verschärfung des Jugendstrafrechts durch Herabsetzung der Strafmündigkeitsgrenze wird abgelehnt. Dem entspricht der einstimmige (!) Beschluss der Justizministerkonferenz vom 1./2.6.2006.

- Die familiengerichtlichen Möglichkeiten der Reaktion auf Gefährdungen des Kindeswohls werden durch gesetzliche Klarstellungen und eine frühzeitigere Inanspruchnahme verbessert.

- $\quad$ Eine Instrumentalisierung des Jugendhilferechts durch genuin jugendstrafrechtliche Interventionen soll ausgeschlossen bleiben.

- Die - ggf. geschlossene - Unterbringung bleibt „ultima ratio“ der Jugendhilfe und ist nur zulässig, „wenn sie zum Wobl des Kindes, insbesondere zur Abwendung einer erheblichen Selbst- oder Fremdgefährdung, erforderlich ist und der Gefahr nicht auf andere Weise, auch nicht durch andere öffentliche Hilfen, begegnet werden kann" (vgl. \$1631b BGB n. F.).

Aufgrund der Koalitionsvereinbarung der Großen Koalition von CDU/CSU und SPD vom 11.11.2005 war im März 2006 vom Bundesjustizministerium eine Arbeitsgruppe eingesetzt worden, die am 17.11.2006 ihren Abschlussbericht vorlegte (der Bericht ist auf der Internetseite des BMJ eingestellt, vgl. www.bmj.de). Der Arbeitsgruppe gehörten insgesamt 23 Experten an (darunter 6 aus dem Bereich des BMJ und 17 aus der Wissenschaft und Praxis des Familien-, Jugendhilfe- und Jugendstrafrechts, u. a. der Verf.). Es wurden zwei Unterarbeitsgruppen zu den Themen (geschlossene) „Unterbringung “ und „Informationsfluss und Zusammenarbeit“ gebildet. $\mathrm{Zu}$ den Beratungen wurden weitere Experten, insbesondere aus der Praxis der Jugendhilfe und Jugendpsychiatrie, einbezogen.

Die Ergebnisse mündeten in einen Gesetzentwurf der Bundesregierung vom 24.10.2007 (BT-Drs. 16/6815) und im April $2008 \mathrm{zu}$ einem entsprechenden Beschluss des Bundestages zur Reform des Familienrechts und dabei vor allem der zentralen Vorschriften der \\1666, 1631b BGB. Das Gesetz ist - wie erwähnt - im Juli 2008 in Kraft getreten.

Den Vorschlägen der Arbeitsgruppe lagen umfassende Problemanalysen, u. a. auch zur Heimunterbringung, zugrunde, die vor allem auf Untersuchungen des Deutschen Jugendinstituts (DJI) gestützt werden konnten. Danach spielt die geschlossene Heimunterbringung in der bundesdeutschen Praxis nur eine sehr untergeordnete Rolle. Am 31.12.2000 befanden sich im Rahmen der Hilfen zur Erziehung 69.723 junge Menschen in der Heimerziehung, davon 10.164 mit familienrechtlicher Genehmigung. Für die geschlossene Unterbringung standen mit Stand Oktober 2006 in Deutschland 260 Plätze 
zur Verfügung, d. h. die geschlossene Heimunterbringung betrifft ca. 0,3\% der Heimerziehung insgesamt. Von den 260 Plätzen waren 91 Plätze nur fakultativ geschlossen, so dass man von weniger als 200 tatsächlich geschlossenen Heimplätzen ausgehen kann. Bezogen auf die jährlich etwa 10.000 familiengerichtlich genehmigten Heimunterbringungen machen die 200 Fälle von Unterbringungen in geschlossenen Einrichtungen lediglich 2\% aus (Hoops/Permien 2006, S. 24). Hinzu kommt, dass die starre Unterscheidung ,geschlossen/ offen " heutzutage so nicht mehr zutrifft. Oft sind die Grenzen fließend, wenn Plätze als „teilgeschlossen“ oder temporär geschlossen genutzt werden (z. B. sog. Time-out-Bereiche). Daher ist die Polarisierung früherer Debatten zur geschlossenen Unterbringung in dieser Hinsicht als überholt anzusehen. In jedem Fall sind aber die nationalen und internationalen menschenrechtlichen Standards zu beachten (vgl. zu den Europäischen Grundsätzen für Jugendliche unter Freiheitsentzug und ambulanten Maßnahmen Dünkel NK 3/2008, S. 102 ff.).

Interessant war bei den Analysen des DJI, dass die 260 (ggf. nur fakultativ) geschlossenen Plätze regional sehr unterschiedlich verteilt sind. Die 260 Heimplätze entfallen auf 19 Einrichtungen in 6 Bundesländern. Bayern verfügt über 82 , Baden-Württemberg über 60 , Nordrhein Westfalen über insgesamt 48 Plätze (Stand: Oktober 2006). Rheinland-Pfalz (28 Plätze), Hamburg (6 Plätze) und Brandenburg (32 Plätze, alle fakultativ geschlossen) als einziges ostdeutsches Bundesland waren die anderen Bundesländer mit geschlossenen Einrichtungen, während alle übrigen 10 Bundesländer über keine derartigen Einrichtungen verfügten (vgl. Arbeitsgruppe ... 2006, S. 37 ff.). Dementsprechend kamen nach einer Umfrage des DJI 87\% der Heimbewohner aus den Bundesländern mit geschlossenen Einrichtungen (37\% allein aus Bayern). Auf die 10 Bundesländer ohne geschlossene Heime entfielen dagegen nur 13\% der Heiminsassen (Hoops/Permien 2006, S. 25). Dies spricht dafür, dass die Schaffung solcher Einrichtungen auch eine gewisse Sogwirkung der Belegung verursacht (schon allein, um die teueren Heimplätze wirtschaftlich kostendeckend betreiben zu können; der Tagessatz der Unterbringung betrug durchschnittlich $260,-€)$. Im Übrigen verdeutlichen die Arbeiten des DJI, dass - abgesehen von Fällen akuter Selbst- und Fremdgefährdung - in der Praxis der Jugendhilfe und Jugendpsychiatrie keine klaren Indikationen für eine geschlossene Unterbringung in der ein oder anderen Form feststellbar sind (Hoops/Permien 2006, S. 31 ff.; Permien 2006, S. 116). Daher ist die Konsequenz, eine gute Kooperation beider Systeme zu fordern, richtig.

Im Einzelnen bringt das Gesetz folgende Neuerungen (vgl. den Gesetzentwurf der Bundesregierung v. 24.10.2007, BT-Drs. 16/6815):

\1666 Abs. 1 BGB hat folgende Fassung erhalten: „Wird das körperliche, geistige oder seelische Wohl des Kindes oder sein Vermögen gefährdet und sind die Eltern nicht gewillt oder nicht in der Lage, die Gefahr abzuwenden, so hat das Familiengericht die zur Abwendung der Gefahr erforderlichen Maßnahmen zu treffen. "In der bisherigen Fassung waren als Tatbestandvoraussetzungen der Missbrauch des Erziehungsrechts bzw. das „Versagen der Eltern“ enthalten. Durch den Verzicht auf schwer feststellbare Tatbestandshürden wie „die missbräuchliche Ausübung der elterlichen Sorge“ oder das „unverschuldete Versagen der Eltern“ sollen frühzeitige „niedrigschwellige präventive Maßnahmen“ erleichtert werden, wie z. B. Weisungen an die Eltern, Leistungen der Jugendhilfe in Anspruch zu nehmen. Die Streichung des impliziten Vorwurfs des Erziehungsversagens soll einer darauf basierenden Beeinträchtigung der Kooperationsbereitschaft der Eltern entgegenwirken. Das Familiengericht hat nach
\ 1666 Abs. 1 BGB „die erforderlichen Maßnahmen“ zu treffen. Das Gesetz sieht dazu in Abs. 2a eine Konkretisierung der Maßnahmen wie folgt vor: „Zu den gerichtlichen Maßnabmen nach Abs. 1 gehören insbesondere 1. Gebote, öffentliche Hilfen wie z.B. Leistungen der Kinder- und Jugendhilfe und der Gesundheitsfürsorge in Anspruch zu nehmen, 2. Gebote, für die Einhaltung der Schulpflicht zu sorgen, 3. Verbote, vorübergehend oder auf unbestimmte Zeit die Familienwohnung oder eine andere Wohnung zu nutzen, sich in einem bestimmten Umkreis der Wohnung aufzuhalten oder zu bestimmende andere Orte aufzusuchen, an denen sich das Kind regelmäßig aufhält, 4. Verbote, Verbindung zum Kind aufzunehmen oder ein Zusammentreffen mit dem Kind herbeizuführen, 5. die Ersetzung von Erklärungen des Inhabers der elterlichen Sorge, 6. die teilweise oder vollständige Entziehung de elterlichen Sorge."

Dadurch soll den Familiengerichten und Jugendämtern die Bandbreite möglicher Maßnahmen - auch unterhalb der Schwelle der Sorgerechtsentziehung - verdeutlicht werden.

Das Gesetz erweitert ferner bei den Verfahrensvorschriften die Möglichkeit der „Erörterung der Kindeswohlgefährdung“ im Rahmen eines richterlichen, die Eltern zur Teilnahme verpflichtenden Erörterungstermins (vgl. \50f FGG). Soweit das Familiengericht von Maßnahmen nach $\$ 1666$ BGB absieht, soll es verpflichtet werden, i. d. R. nach drei Monaten, seine Entscheidung zu überprüfen (vgl. $\$ 1696$ Abs. 3 BGB). Dadurch soll verhindert werden, dass Eltern nach einem für sie folgenlosen Gerichtsverfahren nicht mit dem Jugendamt kooperieren und ihrem Kind dadurch notwendige Maßnahmen vorenthalten. In $\ 50$ e FGG wird das Vorrang- und Beschleunigungsgebot verdeutlicht: „... Verfahren wegen Gefährdung des Kindeswohls sind vorrangig und beschleunigt durchzuführen." $\mathrm{Zu}$ Recht hebt der Deutsche Richterbund in seiner Stellungnahme hervor, dass „die Intensivierung des Schutzes gefährdeter Kinder weniger ein Problem der Gesetzgebung als des Gesetzesvollzugs ist“ (vgl. die Stellungnahme vom Juni 2007 unter www.drb.de/cms/index.php?id=93). Es wird mit anderen Worten vor allem um die Bereitstellung zusätzlicher Personalstellen bei den Familiengerichten und bei den Jugendämtern gehen, um die auch in anderen Bereichen bekannten Vollzugsdefizite abzubauen.

Breiten Raum in den Beratungen der Reform familiengerichtlicher Maßnahmen nahm die Frage der ggf. geschlossenen Unterbringung nach $\ 1631$ b BGB ein. Die Neuregelung lautet nunmehr: „Eine Unterbringung des Kindes, die mit Freiheitsentzug verbunden ist, bedarf der Genehmigung des Familiengerichts. Die Unterbringung ist zulässig, wenn sie zum Wobl des Kindes, insbesondere zur Abwendung einer erheblichen Selbst- oder Fremdgefährdung, erforderlich ist und der Gefahr nicht auf andere Weise, auch nicht durch andere öffentlichen Hilfen, begegnet werden kann. "Damit soll eine Konkretisierung der Voraussetzungen der (ggf. geschlossenen) Unterbringung im Hinblick auf Erfordernisse des Kindeswohls, eine Betonung des Verhältnismäßigkeitsgedankens und des Vorrangs anderer öffentlicher Hilfen erfolgen. "Eine materielle Verschärfung ist damit nicht verbunden“ (vgl. Bt-Drs. 16/6815, Begründung, S. 12). Vor einer Unterbringung ist ein Sachverständigengutachten einzuholen, das bislang in der Regel von einem Arzt für Psychiatrie zu erstellen war. $\$ 70$ e FGG differenziert nunmehr stärker und stellt klar, dass es sich i. d. R. um einen Arzt für Kinder-und Jugendpsychiatrie und -psychotherapie handeln soll. Ferner eröffnet die Vorschrift die Gutachtenpraxis für „in der Heimerziehung ausgewiesene Psychotherapeuten, Psychologen, Pädagogen und Sozialpädagogen" $(\mathbb{S 7 0 \mathrm { e }}$ Abs. 1 S. 3 FGG). 
Besonderer Wert wird auf eine verbesserte Zusammenarbeit von Familiengerichten mit Jugendämtern und anderen Institutionen einschließlich der Schule gelegt. Die Arbeitsgruppe hatte daher vorgeschlagen, $\mathbb{\int}$ 81a SGB VIII wie folgt zu fassen: „Die Träger der öffentlichen Jugendhilfe sollen die Bildung von ständigen Arbeitskreisen mit den Familiengerichten anstreben. In den Arbeitskreisen soll die Zusammenarbeit insbesondere in Fragen der elterlichen Sorge fallübergreifend erörtert und abgestimmt werden. Zu den Arbeitskreisen können andere Institutionen und Personen hinzugezogen werden. "In diesem Zusammenhang hatte die Arbeitsgruppe vorgeschlagen, den Schulen zu ermöglichen, sich u. U. direkt an die Gerichte zu wenden: „In Fällen von Gefährdungen des Kindeswobls wendet sich die Schule in der Regel an das Jugendamt, das ggf. seinerseits die Anrufung des Familiengerichts prüft. Die Arbeitsgruppe weist jedoch ausdrücklich auch auf die Möglichkeit der Schulen hin, sich im Einzelfall, z. B. bei sog. Schulverweigerern, unmittelbar an das Familiengericht zu wenden, wenn andernfalls ein erforderliches schnelles Eingreifen des Familiengerichts nicht gewährleistet ist. Die Arbeitsgruppe appelliert an die Schulverwaltungen, dass diese ibre Schulleiter und Lehrer über die rechtlichen Möglichkeiten und die verschiedenen Ansprechpartner bei Kindeswohlgefährdungen informieren (vgl. Abschlussbericht der Arbeitsgruppe „Familiengerichtliche Maßnabmen ... “ 2006, S. 10). Ferner wurde von der Arbeitsgruppe auch die Verpflichtung der Familien- und Vormundschaftsrichter zur regelmäßigen Fortbildung gefordert. Letztere Vorschläge wurden im Gesetzentwurf der Bundesregierung zwar erwähnt, jedoch in einem Bundesgesetz wegen fehlender Gesetzgebungskompetenz als nicht umsetzbar bezeichnet (vgl. Bt-Drs. 16/6815, Begründung, S. 10).

Die Gesetzesvorschläge sind insgesamt durchweg positiv aufgenommen worden und es gab erfreulicherweise keinerlei grundlegenden Dissens in den Gesetzesberatungen (s. o.). Ein tatsächlich verbesser- ter Schutz des Kindeswohls wird nicht von diesem Gesetz, sondern von dessen Vollzug abhängen und hierfür bedarf es einer Umkehr der Politik der vergangenen Jahre, Stellenkürzungen bei den Kommunen (Jugendamt) und den Familiengerichten vorzunehmen.

Die Bedeutung des Gesetzes liegt aus der Perspektive des Jugendstrafrechts vor allem darin, was „verhindert“ wurde. So wurde - letztlich im Konsens auch mit Bayern - der frühere Vorschlag, die Erziehungsmaßregeln des $\$ 10$ JGG ins Jugendhilfe- bzw. Familienrecht zu transferieren, nicht weiter verfolgt. Dementsprechend sind zivilrechtliche Zwangsmaßnahmen wie die sog. Anti-Social-Behaviour-Orders in England (vgl. hierzu Horsfield in NK 2006, S. 42 ff.) in Deutschland kein Thema. Auch jegliche Diskussion um die Herabsetzung des Strafmündigkeitsalters ist damit vom Tisch. Daran sollte man in den nächsten Wahlkämpfen erinnern, wenn wild gewordene Politiker entsprechende Forderungen wieder aufgreifen sollten. Das Jugendhilferecht bzw. die Familiengerichte verfügen nunmehr über ein sachgerechtes Instrumentarium, um auch in schwierigen Fällen der Gefährdung des Kindeswohls angemessen und effektiv zu reagieren.

\section{Literatur:}

Arbeitsgruppe „Familiengerichtliche Maßnahmen bei Gefäbrdung des Kindeswobls" (2006): Abschlussbericht vom 27.11.2006. Berlin: Bundesministerium der Justiz, www.bmj.bund.de.

Hoops, S., Permien, H. (2006): „Mildere Maßnahmen sind nicht möglich“. Freiheitsentziehende Maßnahmen nach $\$ 1631$ b BGB in Jugendhilfe und Jugendpsychiatrie. München: Deutsches Jugendinstitut.

Permien, H. (2006): Indikationen für geschlossene Unterbringung in der Praxis von Jugendhilfe und Jugendpsychiatrie. Recht \& Politik 24, S. 111118.

Prof. Dr. Frieder Dünkel lehrt Kriminologie an der Universität Greifswald und ist Mitherausgeber dieser Zeitschrift.

\section{Das wahre Leben pocht zwischen den Idealtypen}

\section{Über die „Methode der idealtypisch-vergleichenden Einzelfallanalyse“ (MIVEA) in der Praxis der Strafrechtspflege}

Jürgen Oetting

\begin{abstract}
Sie ist weder in Tiegeln noch Tuben zu haben und auch eine signifikante Wirkung auf die Haut ist nach Anwendung keinesfalls bewiesen. Dennoch ließ ein mächtiger Pharmakonzern den Markenschutz für MIVEA juristisch verhindern. Klingt ja auch arg nach dem Namen einer bekannten Körperpflegekollektion, hat aber mit Kosmetik überhaupt nichts zu tun. MIVEA steht für die „Methode der idealtypisch-vergleichenden Einzelfallanalyse " und ist ein kriminologisches Diagnoseinstrument.
\end{abstract}

Die Bezeichnung „Methode der idealtypisch-vergleichenden Einzelfallanalyse“ taucht erstmals in Hans Göppingers Buch „Angewandte Kriminologie“ (1985) auf, in dem er das Beurteilungsverfahren als Ergebnis einer qualitativen (Zweit-)Auswertung der „Tübinger Jungtäter-Vergleichsuntersuchung “ (TJVU) präsentiert. Das Label MIVEA wurde später von Michael Bock erfunden. Bock war mit seinen profunden Kenntnisse der weberschen Idealtypenbildung erheb- lich an der Entwicklung der Methode beteiligt (grundlegend Bock 1984). Im MIVEA-Zusammenhang ist Bock sozusagen der behutsam-aktualisierende Sachwalter der Göppinger-Lehre. Zudem ist er nach dem Tode Alexander Böhms einziger Herausgeber des großen Göppinger-Lehrbuchs (Göppinger 2008).

MIVEA füllt die Lücke zwischen den weit verbreiteten intuitiven Beurteilungsverfahren in der juristischen, strafvollzuglichen und (sozial-)pädagogischen Praxis und der auf klinischem Spezialwissen beruhenden psychiatrisch/psychologischen „Gutachterei“. MIVEA ist von Menschen erlern- und anwendbar, die keine der sogenannten „Bezugswissenschaften“ der Kriminologie studiert haben - von Akademikern aber durchaus auch (Fortbildungsangebote unter www.mivea.de) Gerade für Vertreter unterschiedlicher Berufsgruppen mit unterschiedlicher wissenschaftlicher oder nichtwissenschaft- 